\title{
Systematic Multidimensional Quantification of Nanoscale Systems From \\ Bimodal Atomic Force Microscopy Data
}

\author{
Chia-Yun Lai, Sergio Santos, Matteo Chiesa
}

Laboratory for Energy and NanoScience (LENS), Institute Center for Future Energy (iFES), Masdar Institute of Science and Technology, Abu Dhabi, UAE 


\section{Contents}

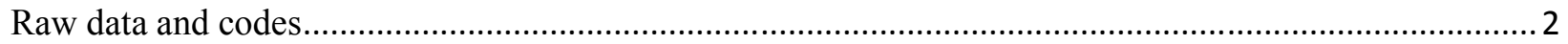

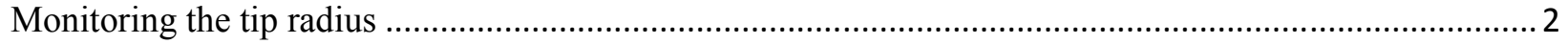

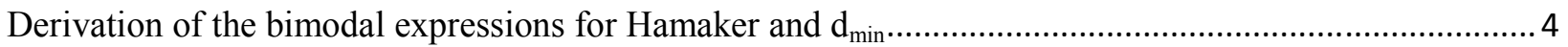

Derivation of main expressions in the text for the van der Waals forces.................................................. 12

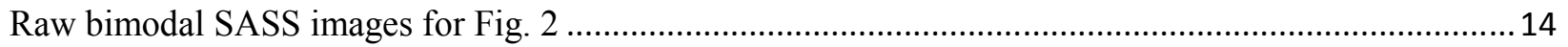

Expressions derived from raw images and cantilever parameters....................................................... 15

Table of $a, b$, and $c$ values for $E^{*}$ (Elastic modulus) in SASS .................................................................17

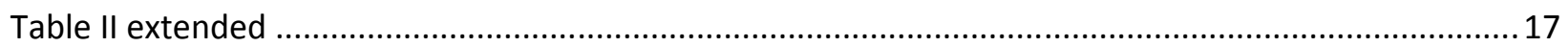

\section{Supplementary}

\section{Raw data and codes}

All raw data and codes (produced via Matlab, Python, the $\mathrm{R}$ language/R studio and $\mathrm{C}$ ) can be found at

https://github.com/bimodalSASS. A Readme.md file is also provided at the site with instructions of how to reproduce all the processed data and figures in this article from the raw data. Matlab, python and $\mathrm{R}$ should be installed in the system before running the scripts and python should be callable under Windows, Linux environments or other.

\section{Monitoring the tip radius}


All the experiments have been carried out with a Cypher AFM from Asylum Research and standard OLYMPUS cantilevers (AC160TS -40 N/m, and AC240TS - 2 N/m). Since it is wellknown that the tip radius $R$ significantly affects the interaction force between the tip and the surface $^{1}$, R was constantly monitored in situ during the experiments. The initial value of $\mathrm{R}$ was assumed to agree with the nominal values provided by the manufacturer, i.e. $\mathrm{R} \approx 10 \mathrm{~nm}$.

The experimental steps to take AFM-APD curves are:

1) A sample was mounted for standard AFM (Cypher AFM from Asylum Research) data acquisition.

2) A new AFM cantilever (OLYMPUS AC160TS with $k=40 \mathrm{~N} / \mathrm{m}$ and $\mathrm{Q}$ factor $\approx 500$ or OLYMPUS AC240TS with $\mathrm{k}=2 \mathrm{~N} / \mathrm{m}$ and $\mathrm{Q}$ factor $\approx 100$ ) was mounted on the AFM cantilever holder.

3) The value of $\mathrm{R}$ was monitored ${ }^{1}$ by acquiring standard ${ }^{2}$ APD curves and these were used to compute the critical amplitude ${ }^{3} \mathrm{~A}_{\mathrm{c}}$ value in raw Volt units ${ }^{1}$. The initial value of $\mathrm{R}$ was assumed to agree with the nominal values provided by the manufacturer, i.e. $\mathrm{R} \approx 10 \mathrm{~nm}$. Provided $\mathrm{A}_{\mathrm{c}}$ did not change for a given sample during the experiments, we assumed that $\mathrm{R}$ remained constant. 4) Approximately 1000-2000 APDs were acquired immediately after computing the value of R for each tip.

5) The raw APD curves were then converted into $F_{\text {ts }}$ versus distance $d$ profiles as those presented in Figs. S1a for HOPG and S1b for PFDA. In Fig. S1 the raw data is shown in red dots and the 
Hamaker fits in blue lines. Each APD produces an $\mathrm{F}_{\mathrm{ts}}$ versus $\mathrm{d}$ profile. We defined $\mathrm{d}=0$ at minima in $\mathrm{F}_{\mathrm{ts}}$, i.e. when $\mathrm{F}_{\mathrm{ts}}$ coincides with the force of adhesion $\mathrm{F}_{\mathrm{AD}}$.

\section{Derivation of the bimodal expressions for Hamaker and $d_{\min }$}

\section{A General Virial expression for the van der Waals force}

This data is reproduced from Ref. $4^{4}$ The bimodal theory ${ }^{5-9}$ states that the virial V for modes 1 and 2 can be obtained directly from observables via

$V_{m}\left(d_{\text {min }}\right) \equiv \frac{1}{T} \oint F_{t s} z_{m} d t=-\frac{A_{m}\left(d_{\min }\right) k_{m} A_{0 m}}{2 Q_{m}} \cos \phi_{m}\left(d_{\text {min }}\right)$

By combining (S1) and (3), where (3) is the model for the force employed in this work in the attractive regime as described in the main text, it can be shown ${ }^{10}$ that

$V_{1}\left(H, d_{\min }\right)=\frac{1}{T} \oint-\frac{R H}{6 d^{2}} z_{1} d t \approx \frac{R H}{6 A_{1}}\left[\left(\frac{d_{\min }+A_{1}}{A_{1}}\right)^{2}-1\right]^{-3 / 2}$

Combining (S1) and (S2) one equation with 2 unknowns, i.e. $\mathrm{d}_{\min }$ and $\mathrm{H}$, results.

A second equation can be derived from the second mode Virial. On the other hand, the second virial should be expressed in terms of the tip position of the first mode $z_{1}$ in order to make the calculations tractable. This simplification was proposed by Kawai et al. ${ }^{11}$ and the approximations discussed in detail by Aksoy and Atalar ${ }^{8}$ and others ${ }^{7}, 12$. Assuming that during a full first mode 
cycle the derivative is an even function of position, the simplifications proposed in the literature, assuming the model in (3), are equivalent to

$V_{2}\left(H, d_{\min }\right)=\frac{1}{T} \oint-\frac{R H}{6 d^{2}} z_{2} d t \approx \frac{R H}{6 \pi} A_{2}^{2} \int_{-1}^{1} \frac{d u}{\left[d_{\min }+A_{1}(1+u)\right]^{3} \sqrt{1-u^{2}}}$

The above results from combining ${ }^{11,13}$

$\frac{1}{T} \oint \frac{\partial F_{t s}}{\partial z} d t \approx-2 k_{2} \frac{\Delta f_{2}}{f_{02}}$

and $^{6-7}$

$V_{2} \approx-k_{2} A_{2}^{2} \frac{\Delta f_{2}}{f_{02}}$

from which the second mode virial can be rewritten as

$V_{2} \approx \frac{A_{2}^{2}}{2 T} \oint \frac{\partial F_{t s}}{\partial z} d t$

The expression in (S5) results from inserting (3) into (S6) and assuming harmonic motion as usual, i.e. $\mathrm{z} \approx \mathrm{A}_{1} \cos \left(\omega_{1} \mathrm{t}-\phi_{1}\right)$

\section{III.B Solution of second mode virial for the van der Waals force}

The objective is to solve the integral in (S7) that can be written as

$I(a, b)=\int_{-1}^{1} \frac{d u}{[a+b(1+u)]^{3} \sqrt{1-u^{2}}}$ 
where $\mathrm{a}=\mathrm{d}_{\min }$ and $\mathrm{b}=\mathrm{A}_{1}$.

\section{$\underline{\text { Analytical solution of (S9) }}$}

After a few changes of variable the solution of (S9) can be found in close form as

$$
\begin{gathered}
I \approx \frac{\left(2 a^{2}+4 a b+3 b^{2}\right) \operatorname{ArcTan}\left[\frac{4.47 \times 10^{7} \sqrt{a}}{\sqrt{a+2 b}}\right]}{2 a^{5 / 2}(a+2 b)^{5 / 2}}+ \\
-\frac{4.47 \times 10^{7} b\left(8 \times 10^{15} a^{2}+1+a b\left(10^{16}+11\right)+6 b^{2}\right)}{2 a^{2}(a+2 b)^{2}\left(a+2 \times 10^{15} b\right)^{2}}
\end{gathered}
$$

Hereafter equation (S8) is considered the analytical solution of (S7). Since (S8) is cumbersome, an approximation is sought.

\section{Approximate solution of (S10)}

Considering that

$\operatorname{ArcTan}\left[\frac{4.47 \times 10^{7} \sqrt{a}}{\sqrt{a+2 b}} \approx 1.571\right.$

Furthermore, from dimensional analysis, and neglecting the second term of (S8)

$$
\frac{1,571\left(2 a^{2}+4 a b+3 b^{2}\right)}{2 a^{5 / 2}(a+2 b)^{5 / 2}}
$$


Furthermore, whenever $b>>a$, it follows that

$$
I \approx \frac{0.833148}{\sqrt{a^{5} b}}
$$

Or

$$
I\left(d_{\min }, A_{1}\right) \approx \frac{0.83}{\sqrt{d_{\min }^{5} A_{1}}}
$$

Hereafter (S11), or equivalently, (S12), is considered the approximate solution to (S7) and (S8) and it is simple enough for the purpose of this work.

\section{Comparison between the numerical integration and the analytic solution (S8) and}

\section{the approximate solution (S11)}

As shown in the example in Fig. S1, the numerical solution coincides with the analytical solution in (S8) with errors smaller than $1 \%$ as compared to numerical integration of (S7). In the figure, the $\mathrm{x}$ axis is $\mathrm{b}=\mathrm{A}_{1}$ and $\mathrm{a}=\mathrm{d}_{\min }$ has been set to $1 \mathrm{~nm}$. The physical implication is that for the range of set point amplitudes explored here, i.e. $1-10 \mathrm{~nm}$, the analytical solution coincides with the numerical integration of (S7). These results have practical use since in attractive bimodal AFM the set point amplitude A1 or Asp lies in such range ${ }^{13-16}$. 


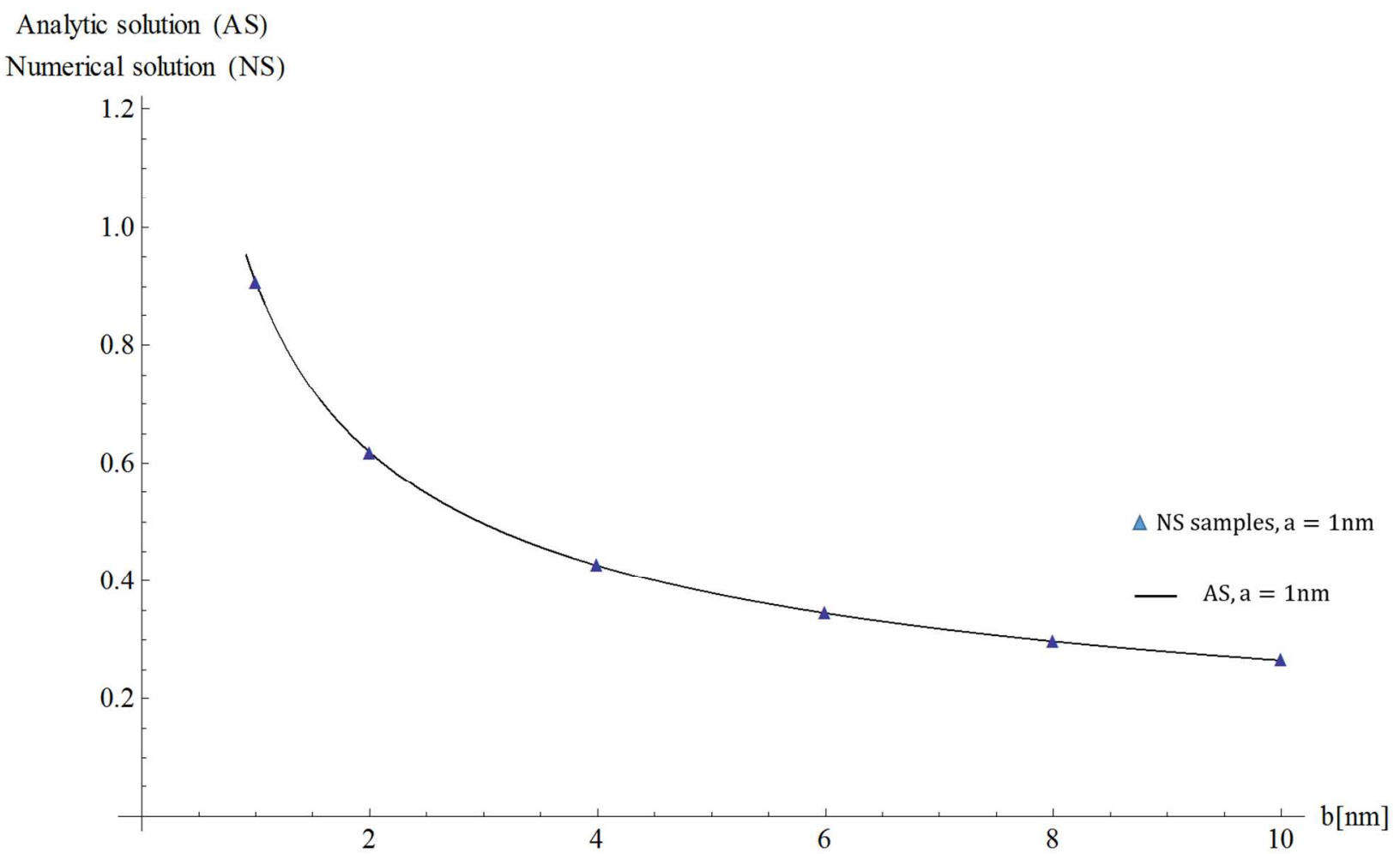

Fig. S1. Comparison between numerical integration of (S7) and the analytical solution in (S8) and as a function of $b$, with $a=1 \mathrm{~nm}$.

The next two figures (Fig. S1 and Fig. S2) show the results of the analytic solution in (S8) that, again, coincides with numerical integration of (S7) with errors smaller than $1 \%$ and the approximate solution in (S11). In Fig. $\mathrm{S} 3 \mathrm{~b} \equiv \mathrm{A}_{1}=10 \mathrm{~nm}$ and $\mathrm{a} \equiv \mathrm{d}_{\min }$ is varied from $0.25 \mathrm{~nm}$ to 1 $\mathrm{nm}$. The approximation in (S11) produces errors smaller than 1\%. An extreme case of operation is presented in Fig. (S6) for which $\mathrm{b} \equiv \mathrm{A}_{1}=0.5 \mathrm{~nm}$ and $\mathrm{a} \equiv \mathrm{d}_{\min }$ varies from 0.25 to $1 \mathrm{~nm}$. In this extreme case errors are still predicted to be smaller than $10 \%$. 
Analytic solution (AS)

Numerical solution (NS)

Approximate solution (AppS)

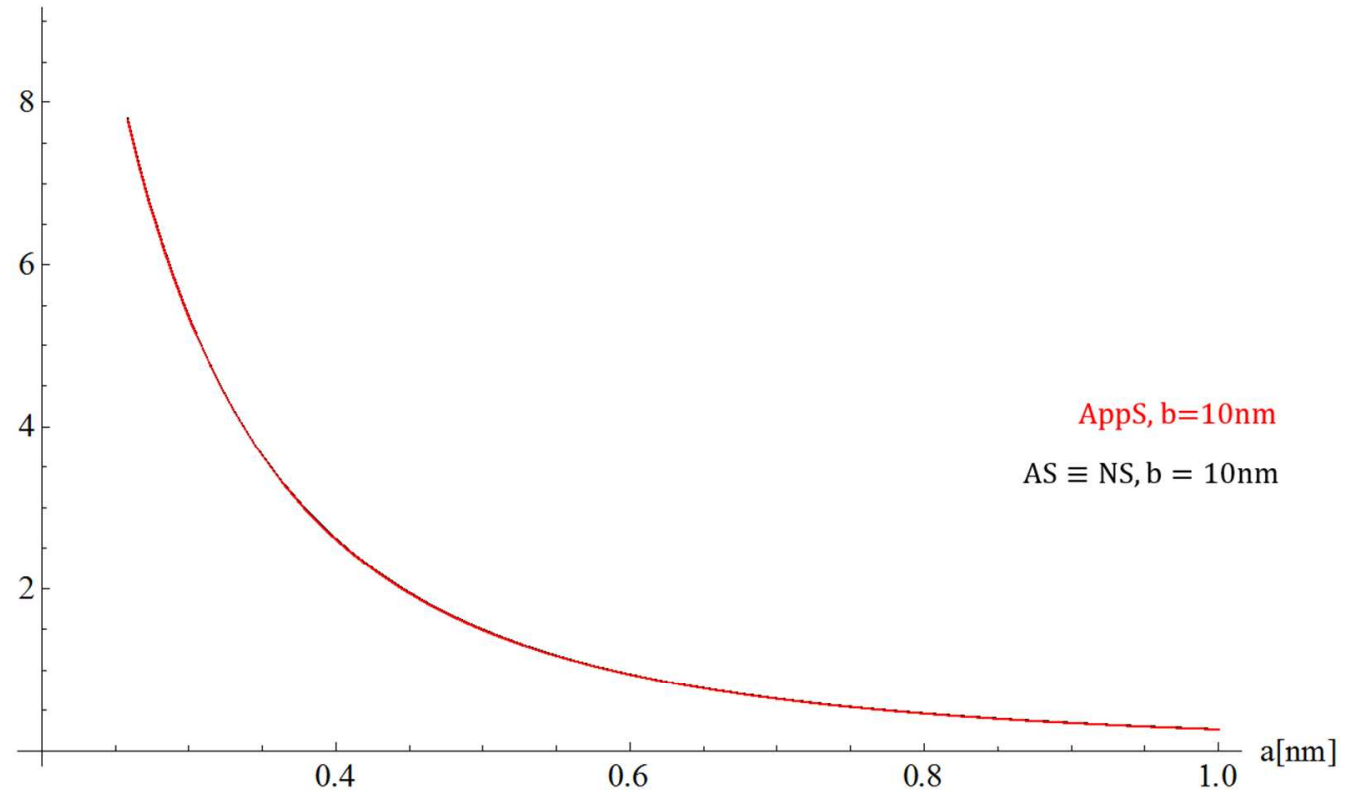

Fig. S2- Analytical-numerical solution compared with the approximation in (S13) for b=10nm.

Analytic solution (AS)

Numerical solution (NS)

Approximate solution (AppS)

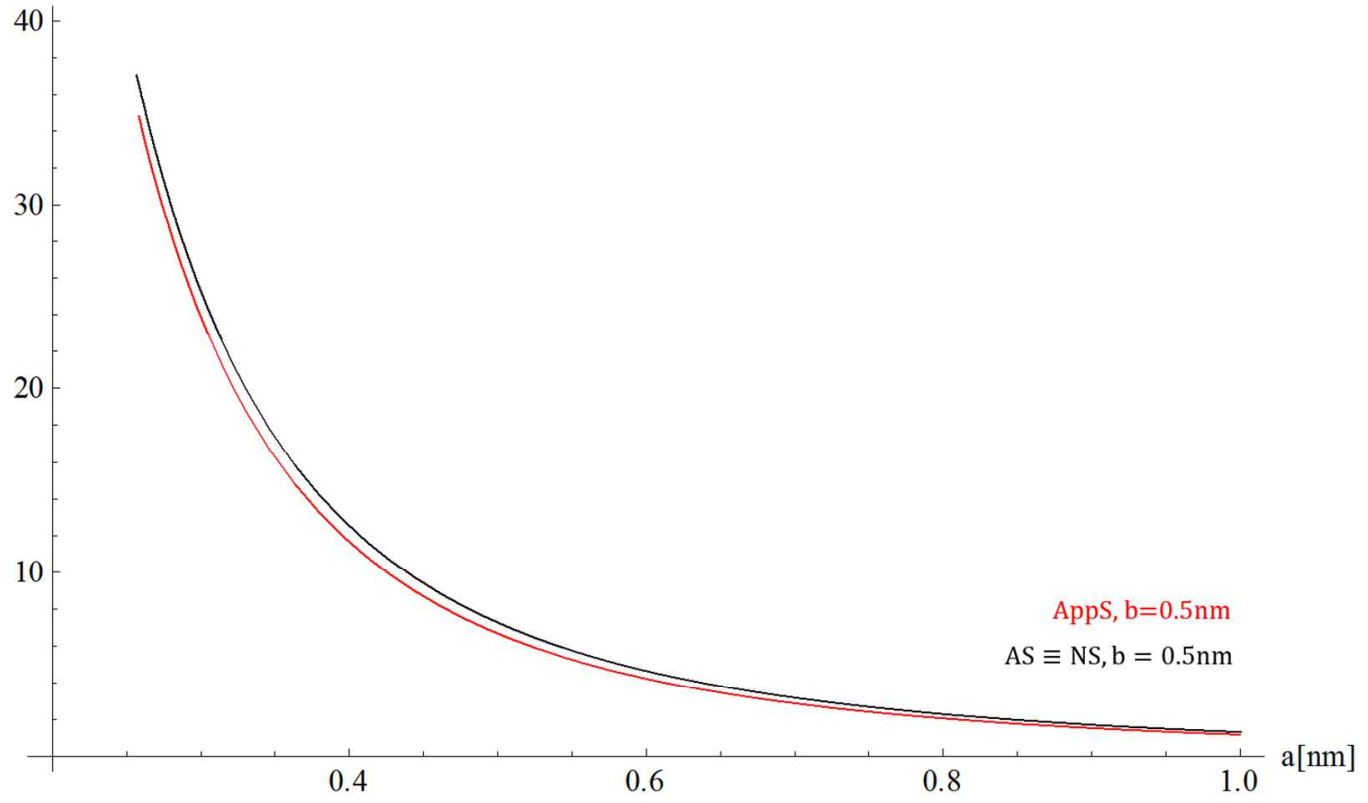

Fig. S3 Analytical-numerical solution compared with the approximated solution given by equation in (S13) with $b=0.5 \mathrm{~nm}$. 
Figs.S3 and S4 show the comparison between the numerical solution as a function of $b$ when is $a=0.2 \mathrm{~nm}$ and $\mathrm{a}=1 \mathrm{~nm}$ respectively.

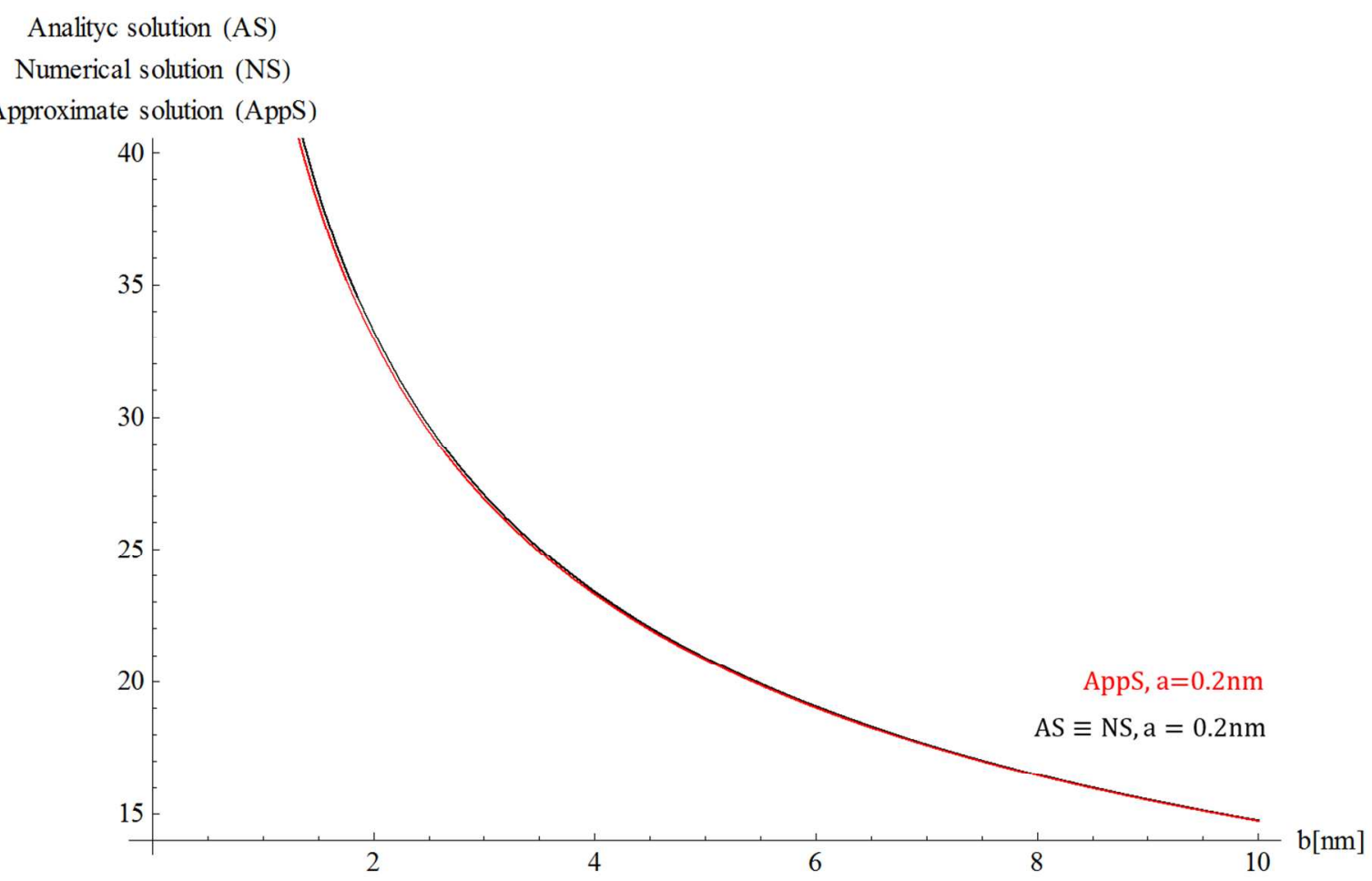

Fig. S4 Analytical-numerical solution compared with the approximated solution given by equation in (S11) with $\mathrm{a}=0.2 \mathrm{~nm}$. 
Analytic solution (AS)

Numerical solution (NS)

Approximate solution (AppS)

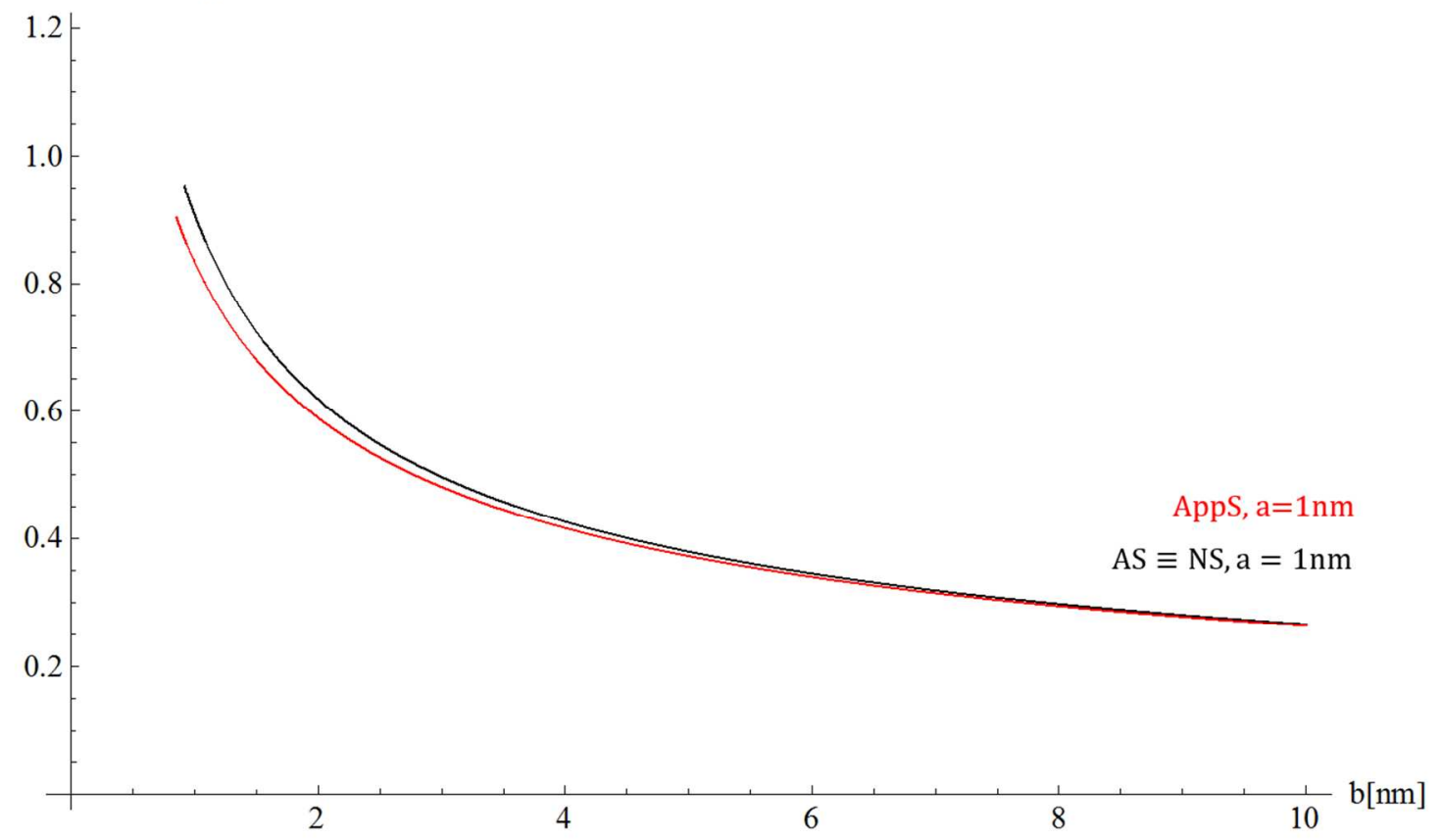

Fig. S5Analytical-numerical solution compared with the approximated solution given by equation in (S11) with a=1 nm.

In summary, the error of the approximation in (S11) is always lower than 5\% except in cases where $a>b$. In such cases the maximum error reaches $11 \%$ the range of parameters explored in the figures above. In principle, provided $a>b$ the error from (S11) tends to zero. The practical result here is that one can employ the approximation in (S11) provided the oscillation amplitude $\mathrm{A}_{1}=\mathrm{A}_{\mathrm{sp}}$, i.e. $\mathrm{b}$, is larger than the minimum distance of approach $\mathrm{d}_{\mathrm{min}}$, i.e. a. In any case, this is true in all our examples since, as corroborated from the force profiles, the decay length of the van der Waals forces is always in the order of 0.5-2 nm while oscillation amplitudes employed in this work for Asp ar4e always larger than $2 \mathrm{~nm}$. 
Derivation of main expressions in the text for the van der Waals forces

Combining (S3) and (S12)

$V_{2}\left(H, d_{\min }\right) \approx \frac{R H}{6 \pi} A_{2}^{2} \frac{0.83}{\sqrt{d_{\min }^{5} A_{1}}}$

Expression (S13) is the second equation necessary with the two unknowns required, i.e. $\mathrm{d}_{\min }$ and

$\mathrm{H}$, that combined with (S2) results in a system of two equations in two unknowns. Solving for

$\mathrm{d}_{\min }$ the solution can be expressed as

$d_{\min }+b d_{\min }^{2 / 3}+c=0$

in accordance with (4) in the main text. The coefficients $b$ and $c$ are found to be

$b=-\left[\frac{3 \pi k_{2} A_{02} Q_{1} \cos \phi_{2}}{0.83 k_{1} A_{01} Q_{2} \cos \phi_{1}}\right]^{2 / 3} \frac{A_{1}}{\left(A_{2}\right)^{2 / 3}}$

$c=2 A_{1}$ 
The zeros of (S14) were found here in Matlab with the help of the standard fzero function and initial values were assumed to lie in the $1 \mathrm{~nm}$ range.

Provided dmin was found for a given pixel in the image, the solution for $\mathrm{H}$ in terms of $\mathrm{V}_{1}$ was trivial $\left(\mathrm{A}_{\mathrm{sp}}=\mathrm{A}_{1}\right)$

$H=-\frac{A_{1}^{2} k_{1} A_{01} \cos \phi_{1}}{R Q_{1}}\left[\left(\frac{d_{\min }+A_{1}}{A_{1}}\right)^{2}-1\right]^{3 / 2}$

and in terms of $\mathrm{V}_{2}$

$H=-\frac{3 \pi k_{2} A_{02} \cos \phi_{2}}{0.83 R Q_{2} A_{2}} \sqrt{d_{\min }^{5} A_{1}}$

When no solution for (S14) were found H was not computed. Errors resulted in some pixels for which dmin was not found. Images for which $10 \%$ of pixels produced errors were discarded. In our case, this resulted mostly when working outside resonance, i.e. this formalism is based on the AFM being operated at the two modal resonances. 
Raw bimodal SASS images for Fig. 2

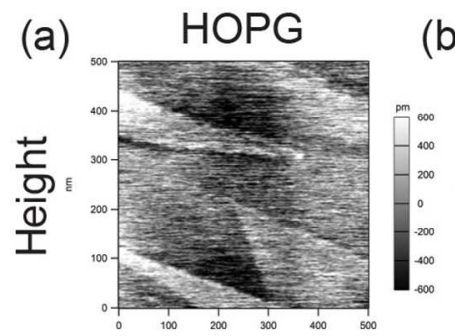

(b)
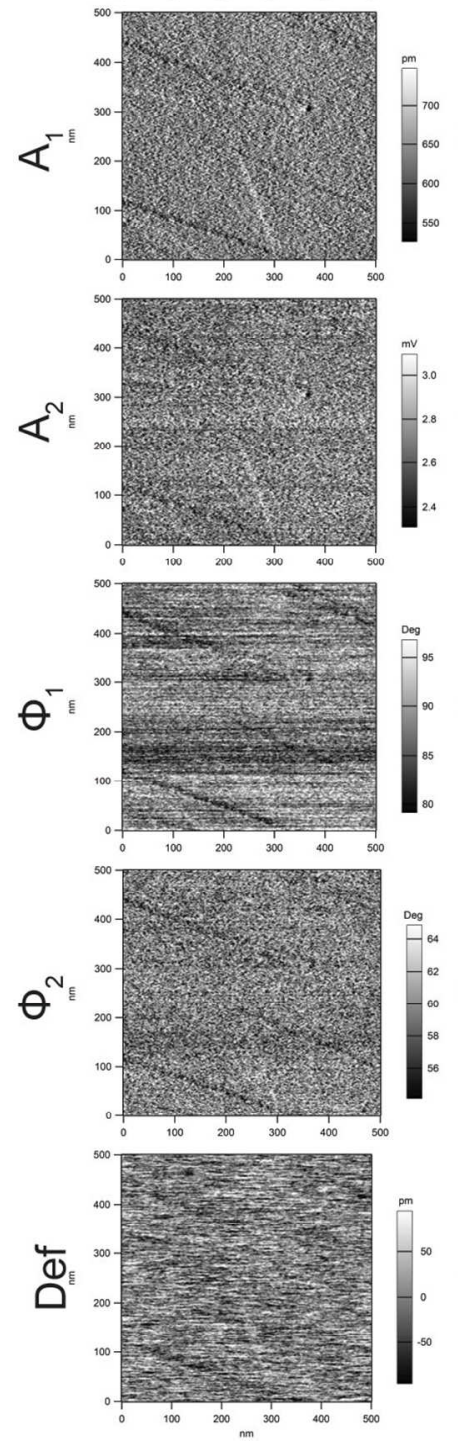

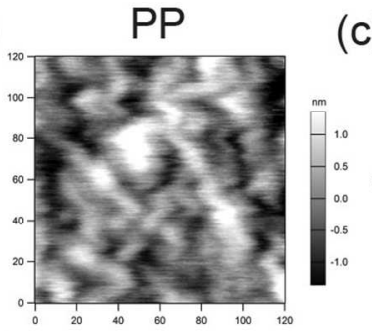

(c)
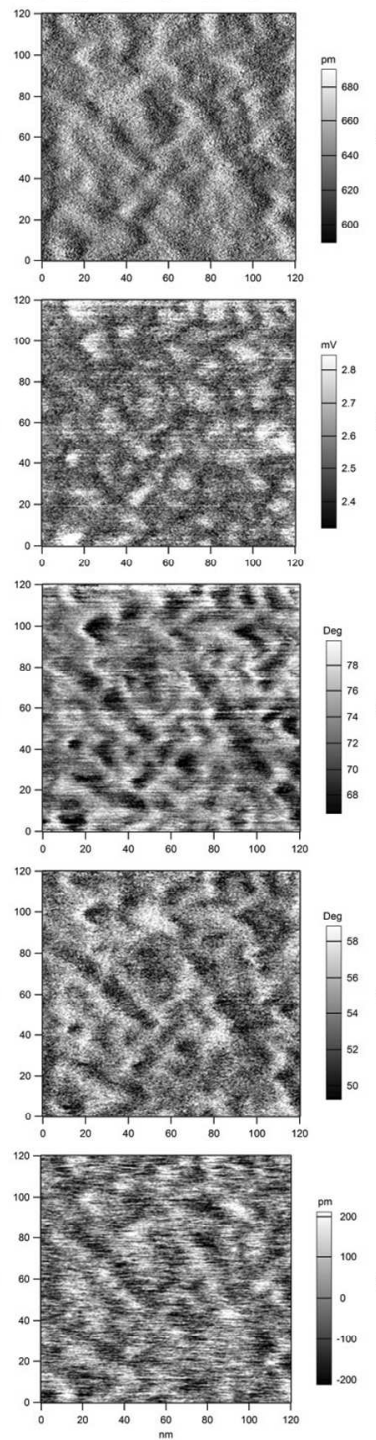
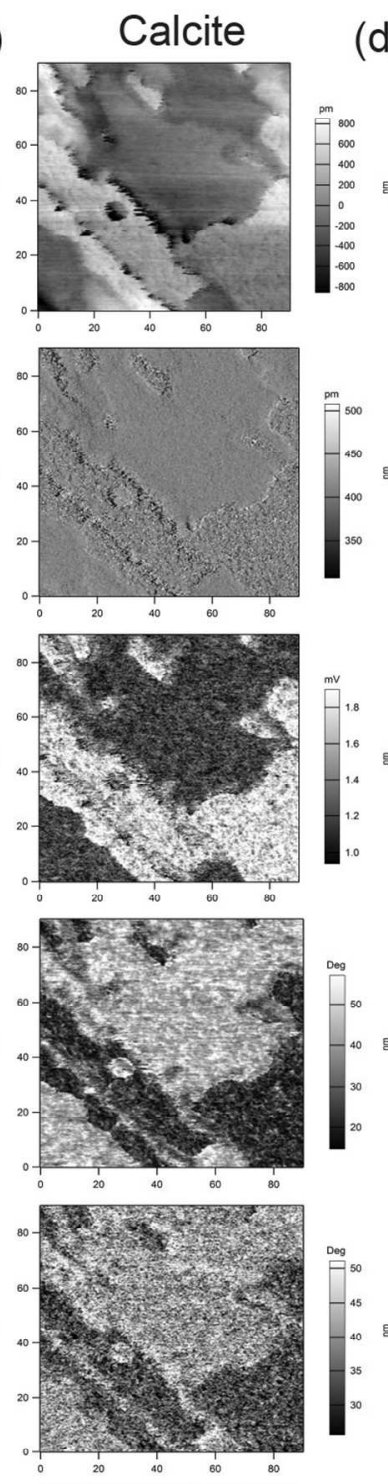

(d)
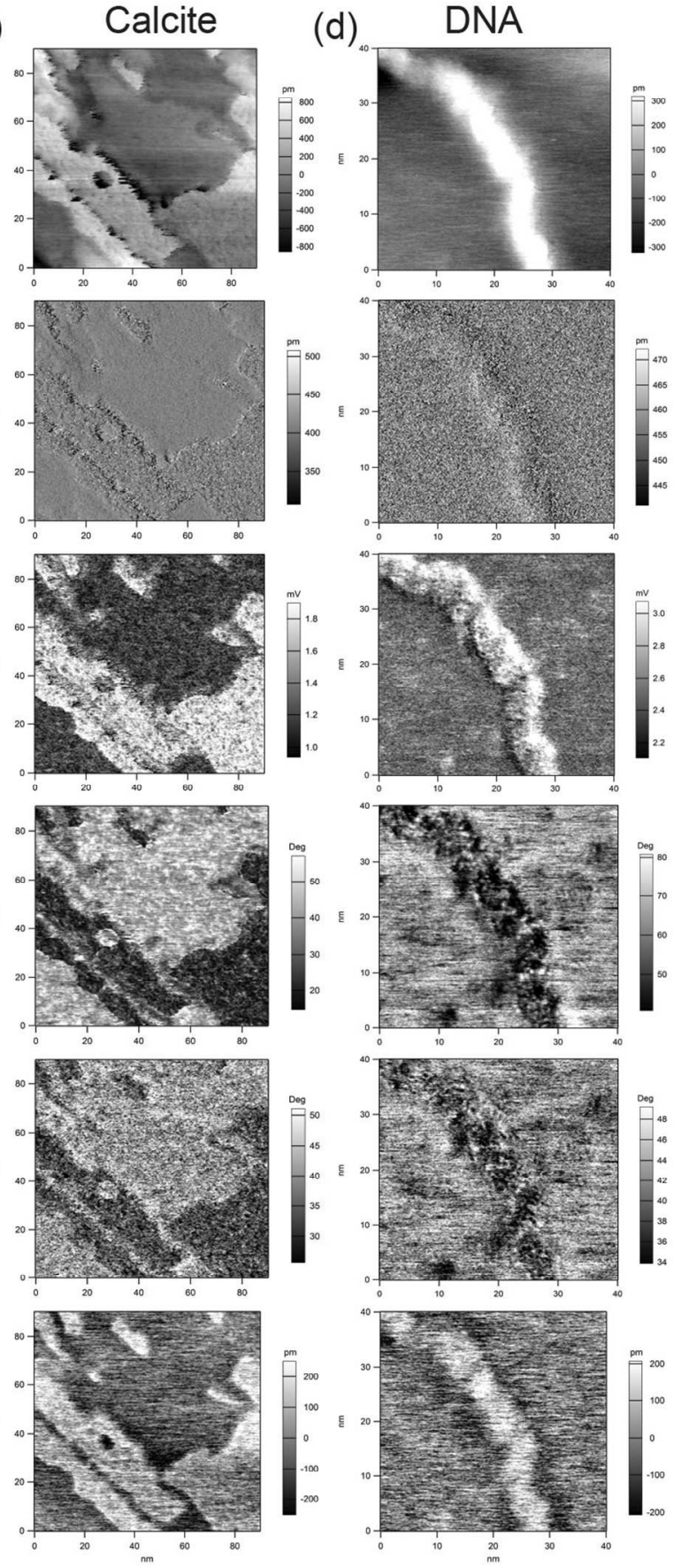
Fig. S6 We note that $A_{1}$ and $A_{2}$ are in the sub-nm range and, in particular, $A 2$ lies in the subAngstrom range as discussed in the main text.

\section{Expressions derived from raw images and cantilever parameters}

The energy transfer from mode to mode can be shown to be written as ${ }^{9}$

$$
E_{T(m)}\left(d_{\min }\right) \equiv \frac{1}{T} \oint F_{t s} d z_{(m)}=-\frac{n \pi k_{(m)} A_{(m)} A_{0(m)}}{Q_{(m)}}\left[\sin \phi_{(m)}\left(d_{\min }\right)-\frac{A_{(m)}}{A_{0(m)}}\right]
$$

where $\mathrm{n}=1$ for the mode 1 or $\mathrm{m}=1$ and $\mathrm{n}$ approximately 6 for mode 2 or $\mathrm{m}=2$.

The kinetic energy for each mode $\mathrm{m}$ is

$$
T_{(m)}\left(d_{\min }\right) \equiv \frac{1}{2} k_{(m)} A_{(m)}^{2}
$$

then $\mathrm{E}_{(\mathrm{m})}$ follows as

$$
E_{(m)}\left(d_{\min }\right) \equiv \frac{E_{(m)} Q_{(m)}}{2 \pi n}+T_{(m)}
$$

The energy dissipated $\mathrm{E}_{\text {dis }}$ is 


$$
E_{d i s}\left(d_{\min }\right) \equiv E_{T(1)}+E_{T(2)}
$$

The modal virial is

$$
V_{(m)}\left(d_{\text {min }}\right) \equiv \frac{1}{T} \oint F_{t s} z_{(m)} d t=-\frac{A_{(m)}\left(d_{\text {min }}\right) k_{(m)} A_{0(m)}}{2 Q_{(m)}} \cos \phi_{(m)}\left(d_{\text {min }}\right)
$$

Examples of these maps for the four model systems in the main text are given in Fig. S7.

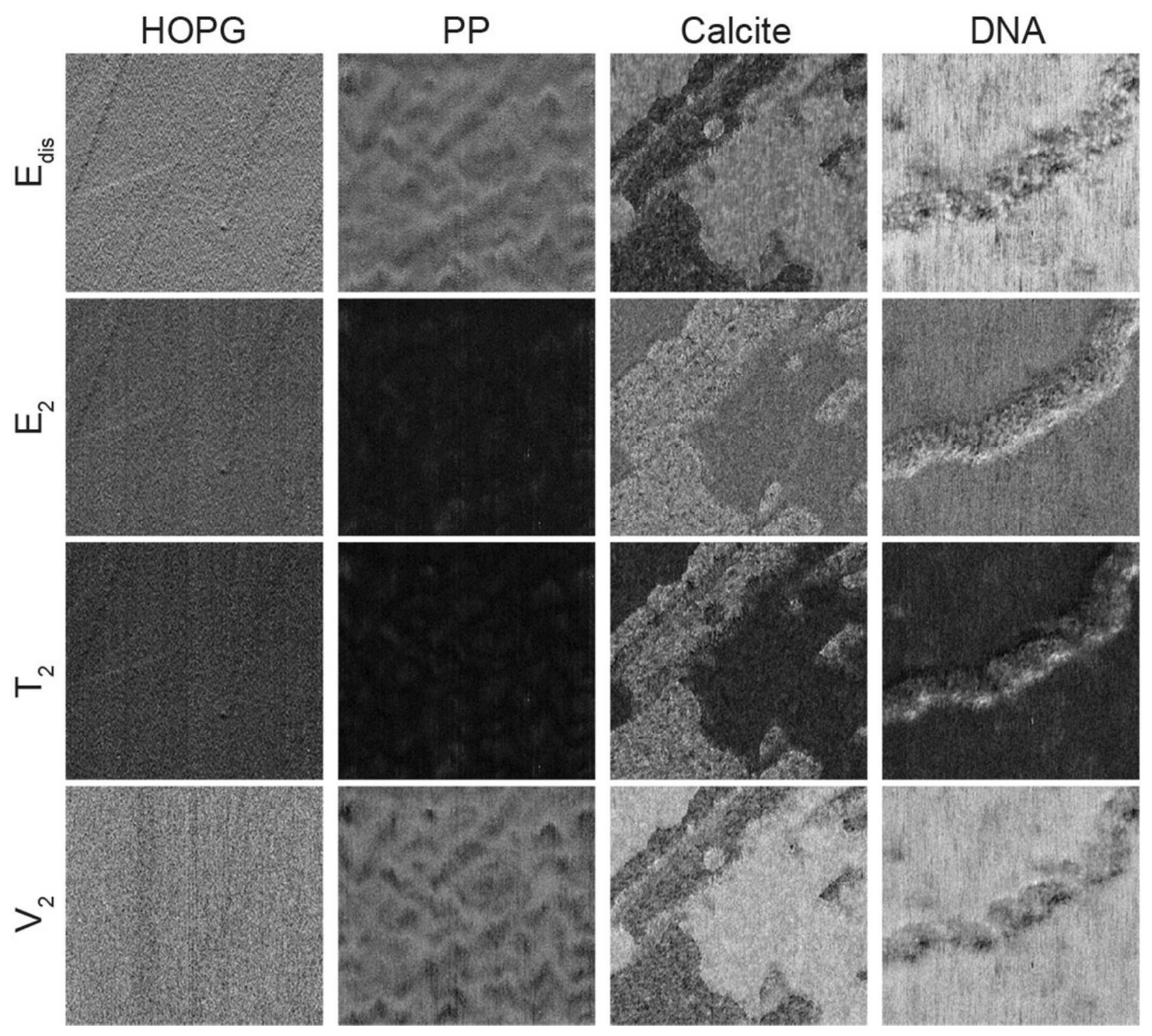

Fig. S7. 
Table of $a, b$, and $c$ values for $\mathbf{E}^{*}$ (Elastic modulus) in SASS

\begin{tabular}{|c|c|c|c|c|c|}
\hline $\mathbf{a}$ & $\mathbf{b}$ & $\mathbf{c}$ & $\mathbf{A}_{\mathbf{0 1}}$ & $\mathbf{A}_{\mathbf{0 2}}$ & $\mathbf{A}_{\mathbf{1}}$ \\
\hline 8 & 0.93 & 0.42 & 8.06 & 0.3 & 0.624 \\
\hline 7.94 & 0.8 & -0.7 & 8.06 & 0.45 & 0.624 \\
\hline 6 & 1.04 & -0.22 & 10.4 & 0.45 & 0.624 \\
\hline 1.055 & 2.01 & 0.66 & 10.4 & 0.3 & 0.624 \\
\hline 1.05 & 2.01 & 0.66 & 10.4 & 0.3 & 0.624 \\
\hline 3.3 & 1.7 & 0.83 & 10.69 & 0.51 & 0.44 \\
\hline 18.38 & 1.05 & -0.74 & 12.48 & 0.9 & 0.39 \\
\hline 23.8 & 1.16 & -0.21 & 12.48 & 1.05 & 0.39 \\
\hline
\end{tabular}

Table II extended 


\begin{tabular}{|c|c|c|c|c|c|c|c|c|}
\hline $\mathrm{E}^{*}[\mathrm{GPa}]$ & $\mathrm{T}_{(1)}\left[{ }^{\circ}\right]$ & $\mathrm{T}_{(2)}\left[{ }^{\circ}\right]$ & $\mathrm{V}_{(1)}[\mathrm{eV}]$ & $\mathrm{V}_{(2)}[\mathrm{eV}]$ & $\mathrm{E}_{\mathrm{T}(1)}[\mathrm{eV}]$ & $\mathrm{E}_{\mathrm{T}(2)}[\mathrm{eV}]$ & $\mathrm{E}_{(1)}[\mathrm{eV}]$ & $\mathrm{E}_{(2)}[\mathrm{eV}]$ \\
\hline 0.10 & 3.06 & 8.68 & -0.48 & -32.01 & 16.60 & -20.35 & 3.32 & 6.74 \\
\hline 0.15 & 3.06 & 7.09 & -0.48 & -28.92 & -32.90 & 29.65 & 2.53 & 9.92 \\
\hline 0.23 & 3.06 & 5.45 & -0.48 & -25.72 & -45.44 & 42.73 & 2.33 & 9.54 \\
\hline 0.34 & 3.06 & 4.40 & -0.48 & -24.10 & 43.34 & -45.66 & 3.75 & 0.04 \\
\hline 0.51 & 3.06 & 3.52 & -0.48 & -22.00 & 100.52 & -102.34 & 4.66 & -6.25 \\
\hline 0.76 & 3.06 & 2.54 & -0.48 & -18.83 & 111.20 & -112.37 & 4.83 & -8.19 \\
\hline 1.14 & 3.06 & 1.51 & -0.48 & -14.49 & 31.00 & -31.53 & 3.55 & -1.50 \\
\hline 1.71 & 3.06 & 0.99 & -0.48 & -11.23 & -71.66 & 71.57 & 1.92 & 7.82 \\
\hline 2.56 & 3.06 & 0.75 & -0.48 & -8.88 & -147.34 & 147.55 & 0.71 & 14.84 \\
\hline 3.84 & 3.06 & 0.64 & 0.48 & -7.07 & -185.64 & 199.06 & 0.10 & 19.65 \\
\hline 5.77 & 3.06 & 0.59 & 0.48 & -5.61 & -153.00 & 231.90 & 0.62 & 22.73 \\
\hline 8.65 & 3.06 & 0.55 & 0.48 & -4.43 & -133.84 & 251.23 & 0.93 & 24.54 \\
\hline 12.97 & 3.06 & 0.52 & 0.48 & -3.47 & -124.22 & 260.99 & 1.08 & 25.44 \\
\hline 19.46 & 3.06 & 0.49 & 0.48 & -2.67 & -121.24 & 264.11 & 1.13 & 25.71 \\
\hline 29.19 & 3.06 & 0.45 & 0.48 & -2.02 & -122.73 & 262.74 & 1.10 & 25.54 \\
\hline 43.79 & 3.06 & 0.42 & 0.48 & -1.50 & -127.04 & 258.54 & 1.04 & 25.11 \\
\hline
\end{tabular}

Reference

1. Santos, S.; Guang, L.; Souier, T.; Gadelrab, K.; Chiesa, M.; Thomson, N. H., A Method to Provide Rapid in Situ Determination of Tip Radius in Dynamic Atomic Force Microscopy. Rev. Sci. Instrum 2012, 83043707.

2. Ziegler, K. J.; Lyons, D. M.; Holmes, J. D.; Erts, D.; Polyakov, B.; Olin, H.; Svensson, K.; Olsson, E., Bistable Nanoelectromechanical Devices. Appl. Phys. Lett. 2004, 844074.

3. Santos, S.; Guang, L.; Souier, T.; Gadelrab, K. R.; Chiesa, M.; Thomson, N. H., A Method to Provide Rapid in Situ Determination of Tip Radius in Dynamic Atomic Force Microscopy. Rev. Sci. Instrum 2012, 83 043707-043717.

4. Lai, C.-Y.; Perri, S.; Santos, S.; Garcia, R.; Chiesa, M., Rapid Quantitative Chemical Mapping of Surfaces with Sub-2nm Resolution. Nanoscale 2016. 
5. Lozano, J. R.; Garcia, R., Theory of Multifrequency Atomic Force Microscopy. Phys. Rev. Lett. 2008, 100076102.

6. Lozano, J. R.; Garcia, R., Theory of Phase Spectroscopy in Bimodal Atomic Force Microscopy. Phys. Rev. B 2009, 79014110.

7. Herruzo, E. T.; Garcia, R., Theoretical Study of the Frequency Shift in Bimodal Fm-Afm by Fractional Calculus. Beilstein J. Nanotechnol. 2012, 3 198-206.

8. Aksoy, M. D.; Atalar, A., Force Spectroscopy Using Bimodal Frequency Modulation Atomic Force Microscopy. Phys. Rev. B 2011, 83075416.

9. Santos, S., Phase Contrast and Operation Regimes in Multifrequency Atomic Force Microscopy. Appl. Phys. Lett. 2014, 104143109.

10. Paulo, A. S.; Garcia, R., Tip-Surface, Amplitude, and Energy Dissipation in Amplitude-Modulation (Tapping Mode) Force Microscopy. Phys. Rev. B 2001, 64 193411-193414.

11. Kawai, S.; Glatzel, T.; Koch, S.; Such, B.; Baratoff, A.; Meyer, E., Systematic Achievement of Improved Atomic-Scale Contrast Via Bimodal Dynamic Force Microscopy. Phys. Rev. Lett. 2009, 103 220801-220804.

12. Santos, S., Theory of Small Amplitude Bimodal Atomic Force Microscopy in Ambient Conditions arxiv.org/pdf/1401.6687 2014.

13. Martinez-Martin, D.; Herruzo, E. T.; Dietz, C.; Gomez-Herrero, J.; Garcia, R., Noninvasive Protein Structural Flexibility Mapping by Bimodal Dynamic Force Microscopy. Phys. Rev. Lett. 2011, 106198101. 14. Herruzo, E. T.; Perrino, A. P.; Garcia, R., Fast Nanomechanical Spectroscopy of Soft Matter. Nat. Commun. 2014, 5.

15. Kiracofe, D.; Raman, A.; Yablon, D., Multiple Regimes of Operation in Bimodal Afm: Understanding the Energy of Cantilever Eigenmodes. Beilstein J. Nanotechnol. 2013, 4 385-393.

16. An, S.; Solares, S. D.; Santos, S.; Ebeling, D., Energy Transfer Between Eigenmodes in Multimodal Atomic Force Microscopy. Nanotechnology 2014, 25 475701-475711. 